\title{
Relationship between the clinical findings and radiographic severity in Osgood-Schlatter disease
}

This article was published in the following Dove Press journal:

Open Access Journal of Sports Medicine

8 March 2012

Number of times this article has been viewed

\section{Mitsuru Hanada \\ Hiroshi Koyama \\ Masaaki Takahashi \\ Yukihiro Matsuyama}

Department of Orthopedic Surgery, Hamamatsu University School of Medicine, Hamamatsu, Japan
Correspondence: Mitsuru Hanada Department of Orthopedic Surgery, Hamamatsu University School of Medicine, I-20-I Handayama, Hamamatsu, 43I-3192, Japan

$\mathrm{Tel}+8 I 534352299$

Fax +81534352296

Emailmitsuruhanada@gmail.com
Background: Osgood-Schlatter disease (OSD) is one of the common causes of knee pain in active adolescents who play sports. The common age for boys to have OSD is between 12 and 15 years and for girls, between 8 and 12 years. Radiographic studies are helpful in diagnosis and treatment of OSD.

Purpose: We examine the age at onset of OSD in detail and investigate the relationship between clinical findings, radiographic bone morphology, and the severity of OSD in adolescents.

Results: The average age at onset of knee pain was 12 years and 6 months -12 years and 9 months in boys, and 12 years and 1 month in girls. Boys were significantly older than girls at onset. In addition, there were significant relationships between duration from first onset to visit to the clinic, radiographic bone stage, body morphology, and radiographic severity. The patients who delayed their visit to the clinic from the first onset of pain and who were older showed a later bone stage and more radiographic severity grade of OSD. There was significant differences concerning weight and body mass index between severity grade I and III.

Conclusion: For the age at the onset of OSD, the mean age of boys was significantly older than that of girls. The patients at a later bony stage had a higher severity grade. The boys and girls with OSD who had less weight or body mass index showed less severity.

Keywords: Osgood-Schlatter disease, radiographic classification, tibial tuberosity, body morphology

\section{Introduction}

Osgood-Schlatter disease (OSD) is one of the common causes of knee pain in active adolescents who play sports. In 1903, Osgood ${ }^{1}$ and Schlatter ${ }^{2}$ separately described a painful condition of the tibial tuberosity. They suggested that the cause of OSD was a partial traumatic avulsion of the tibial tuberosity at the insertion of the patellar tendon. Afterward, many theories were put forward regarding the etiology of OSD including degeneration of the patellar tendon, aseptic necrosis, and infection. ${ }^{3,4}$ Ogden pathologically investigated OSD, describing it as an avulsion of a portion of the developing ossification center; this avulsion may also occur when the cartilage cells are hypertrophic in the preossification phase..$^{5-7}$ Hirano investigated the progress of OSD from an early stage on magnetic resonance imaging (MRI) and reported that the onset of all OSD was observed to be in the apophyseal stage by MRI. ${ }^{8} \mathrm{~A}$ recent review claimed OSD was caused by traction apophysitis of the tibial tubercle due to repetitive strain on the secondary ossification center of the tibial tuberosity. ${ }^{9}$ Radiographic changes show the irregularity of apophysis, with separation from the tibial tuberosity in early stages and fragmentation in the later stages. The common age of boys with OSD is between the 
ages of 12 and 15 years and the common age of girls with OSD is between the ages of 8 and 12 years. ${ }^{10,11}$ OSD should be suspected in young patients who present with pain, swelling, and tenderness of the tibial tuberosity. Radiographic studies are helpful in diagnosis and treatment of OSD. However, to our knowledge, there is no previous report about the relationship between OSD and body morphology. The aim of this study is to find the age at onset of OSD in detail, to investigate the relationship between clinical and radiographic findings, and to investigate body morphology and the severity of OSD.

\section{Methods}

\section{Subjects}

Seventy-six children and 94 knees were included in the study. The children were diagnosed with OSD with complaints of pain, swelling, tenderness of the tibial tuberosity, and radiographic abnormality of the tibial tuberosity. Clinical evaluation was done by several orthopedic surgeons and radiographic evaluation was done by one orthopedic surgeon. Children who were under 18 years old were included in this study. We investigated the age at onset of OSD, for example, not only "10 years old," but also the details such as " 10 years 6 months old." There were 57 boys ( 73 knees) aged from 10 years 11 months old to 16 years 6 months old (mean, 13 years 4 months old) and 19 girls (21 knees) aged from 9 years 11 months old to 15 years 5 months old (mean, 13 years 4 months old).

\section{Methods}

Each child's history of OSD, in particular the age at onset of knee pain and duration from the onset of pain to the first visit to medical office or hospital for radiography, was examined. Clinical findings were investigated, including pain, swelling, and tenderness of the tibial tuberosity. Body morphology was also investigated, including body weight, height, and body mass index (BMI). The radiographic bone growth stage of the tibial tuberosity was defined by Ehrenborg's classification: stage A is cartilaginous, stage $\mathrm{B}$ is apophyseal, stage $\mathrm{C}$ is epiphyseal, and stage D is bony. ${ }^{12}$ The radiographic severity of OSD was determined by our method as follows: grade I shows upheaval or slight elevation of the tibial tuberosity, grade II shows radiolucency of the tibial tuberosity, and grade III shows fragmentation of the tibial tuberosity or ossicle (Figure 1).

\section{Statistical analysis}

Each item was statistically analyzed for relevance. The ages of boys and girls were compared using the Mann-Whitney test. The relationship between the radiographic bone stage and the radiographic severity was evaluated with the chi-squared test. We evaluated the relationship between the radiographic bone stage and the duration from the onset of pain to the first clinic visit. In addition, sub groups of the duration from the onset of pain to the first visit to medical office or hospital were statistically analyzed using the Mann-Whitney test. The body morphology, height, weight, and BMI were compared to radiographic severity using the Mann-Whitney test.

\section{Results}

The age at onset of knee pain averaged 12 years 6 months old in total, 12 years 9 months for boys and 12 years 1 month for girls. The age of boys at the onset was significantly older than girls with Mann-Whitney test $(P<0.01)$.

For the radiographic bone stage at the initial visit, there was nothing for stage A, 36 knees in stage B, 51 knees in stage $\mathrm{C}$, and seven knees in stage D. For radiographic severity, there were 46 knees at stage I, 22 knees at stage II, and 26 knees at stage III. There was a significant relationship between radiographic bone stage and radiographic severity determined by the chi-squared test $(P<0.01)$ (Table 1$)$.
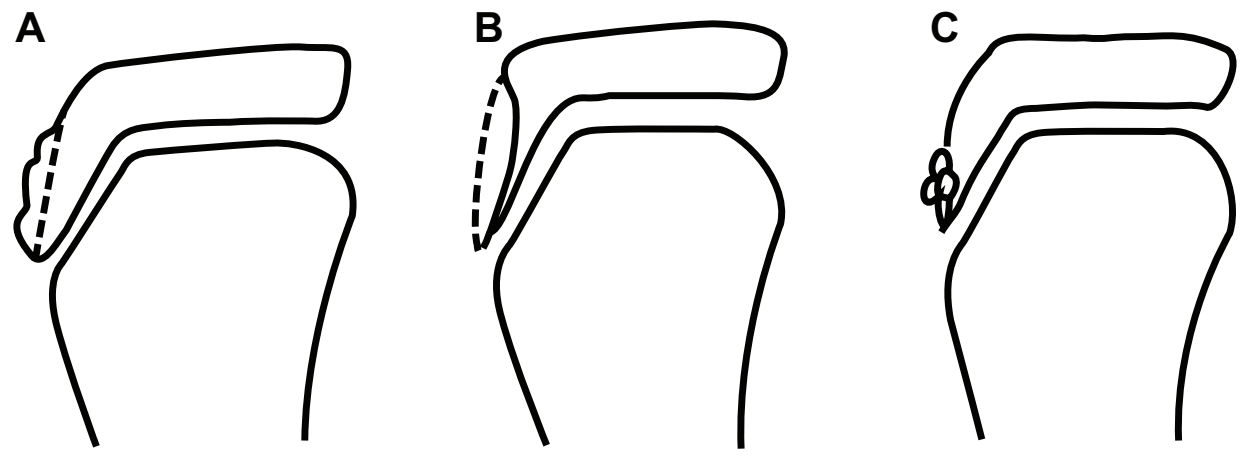

Figure I The severity of Osgood-Schlatter disease in the epiphyseal stage on radiographic findings. (A) Grade I, upheaval or slight elevation of the tibial tuberosity; (B) grade II, radiolucency of the tibial tuberosity; (C) grade III, fragmentation of the tibial tuberosity. 
Table I Relationship between bone growth stage and severity at the first visit to medical office or hospital

\begin{tabular}{llll}
\hline Severity & \multicolumn{3}{l}{ Bone growth stage } \\
\cline { 2 - 4 } & B & C & D \\
\hline I & 20 knees & 26 knees & 0 knees \\
& $(15$ boys, 5 girls $)$ & $(20$ boys, 6 girls $)$ & \\
II & 10 knees & 12 knees & 0 knees \\
& $(9$ boys, I girls) & (II boys, I girl) & \\
III & 6 knees & 13 knees & 7 knees \\
& $(3$ boys, 3 girls $)$ & $(10$ boys, 3 girls $)$ & (5 boys, 2 girls $)$ \\
\hline
\end{tabular}

Note: There was a significant difference between radiographic bone stage and radiographic severity of Osgood-Schlatter disease determined by the chi-squared test $(P<0.01)$.

However, there was no relationship between the clinical findings and radiographic severity (Table 2). There was a significant relationship between the duration from the onset of pain to the first clinic visit and severity determined by the chi-squared test $(P<0.01)$, indicating that patients with a longer period of pain before the first visit to medical office or hospital had greater severity (Table 3 ).

Figure 2 shows that there was no significant difference in the mean height between patients in severity I, II, and III. However, the Mann-Whitney U test $(P<0.01)$ revealed the body weight and BMI were significantly less in patients in severity I than those in severity III.

\section{Discussion}

The common age of boys with OSD is between the ages of 12 and 15 years and the common age of girls with OSD is between the ages of 8 and 12 years. ${ }^{10,11}$ In this study, for the age at onset of OSD, the mean age of boys was significantly older than that of girls, being in agreement with Ehrenborg and Lagergren that the age of occurrence of OSD in boys was greater than in girls. ${ }^{12}$ OSD is known to occur in the apophyseal stage. Hirano et al reported the onset of all OSD were observed to be apophyseal stage by MRI. ${ }^{8}$ We consider that apophyseal stage corresponds to the growth spurt stage; thus the difference of age at onset of OSD between boys and

Table 2 Relationship between clinical findings and severity

\begin{tabular}{llll}
\hline Severity & \multicolumn{3}{l}{ Clinical findings } \\
\cline { 2 - 4 } & Swelling & Tenderness & Osseous upheaval \\
\hline I & 20 knees & 40 knees & 5 knees \\
& $(45 \%)$ & $(90 \%)$ & $(11 \%)$ \\
II & 11 knees & 21 knees & 5 knees \\
& $(50 \%)$ & $(95 \%)$ & $(22 \%)$ \\
III & 12 knees & 12 knees & 6 knees \\
& $(50 \%)$ & $(50 \%)$ & $(25 \%)$ \\
\hline
\end{tabular}

Note: No significant relationship between clinical findings and radiographic severity of Osgood-Schlatter disease determined by the chi-squared test.
Table 3 Relationship between the duration from the onset of pain to the first clinic visit and severity

\begin{tabular}{lllll}
\hline Severity & \multicolumn{3}{l}{$\begin{array}{l}\text { Duration from the onset of pain to first visit to } \\
\text { medical office or hospital }\end{array}$} \\
\cline { 2 - 5 } & $<$ I week & $\begin{array}{l}\text { I week } \\
\sim 3\end{array}$ months & $\begin{array}{l}\text { 3 months } \\
\text { I year }\end{array}$ & $\sim$ I year \\
\hline I & I8 knees & 8 knees & I 3 knees & 2 knees \\
II & 2 knees & I 0 knees & 5 knees & 0 knee \\
III & 2 knees & 8 knees & 5 knees & 8 knees \\
& & $*$ & & $*, * *, * * *$
\end{tabular}

Notes: There was a significant difference between the duration from the onset of pain to the first visit to medical office or hospital and radiographic severity of Osgood-Schlatter disease determined by the chi-squared test $(P<0.01)$; *There was a significant difference by the Mann-Whitney $U$ test (vs " $<$ I week" group: $P<0.01$ ); **There was a significant difference by the Mann-Whitney $U$ test (vs "I week $\sim 3$ months" group: $P<0.01$ ); ***There was a significant difference by the Mann-Whitney $U$ test (vs " 3 months $\sim$ l year" group: $P<0.05$ ).

girls may be the result of differences of the initiation age of growth spurts. There is a correlation between the severity of OSD and the duration from the onset of pain to the first visit to clinic and bone growth stage in the present study. Children with a longer period from the onset of pain to the first visit to a medical office or hospital had pain of greater severity. There were 10 children who had over 1 year's duration between onset of OSD and first visit to a clinic. Eight of these children had OSD with severity grade III ( $80 \%)$. Therefore, boys and girls with OSD should have a medical examination immediately if the symptoms included pain, tenderness, and swelling of tibial tuberosity. This study found all patients at the bony stage had severity grade III, and patients who had a later bone stage showed a higher radiographic severity grade of OSD. Concerning the relationship between body morphology and severity of OSD, there is no significant difference in height between each grade of severity, although there is a significant difference between weight, BMI, and severity of OSD. In this study, there was a significant difference concerning weight and BMI between grades I and III. The boys and girls with less weight or lower BMI showed less severity. However, to our knowledge there has been no previous report on the relationship between OSD and body morphology. Kraus et al reported on an obese adolescent male who suffered from consecutive bilateral fractures of the tibia. ${ }^{13}$ It is possible that increased weight or higher BMI worsens the severity of OSD. Therefore, we must examine more carefully those boys and girls with pain at the tibial tuberosity who are overweight.

In conclusion, for the age at the onset of OSD, the mean age of boys, 12 years 9 months, was significantly older than that of girls, 12 years 1 month. In addition, children at a later bony stage had a higher severity grade while patients with lower weight or lower BMI had a lower severity of OSD. 

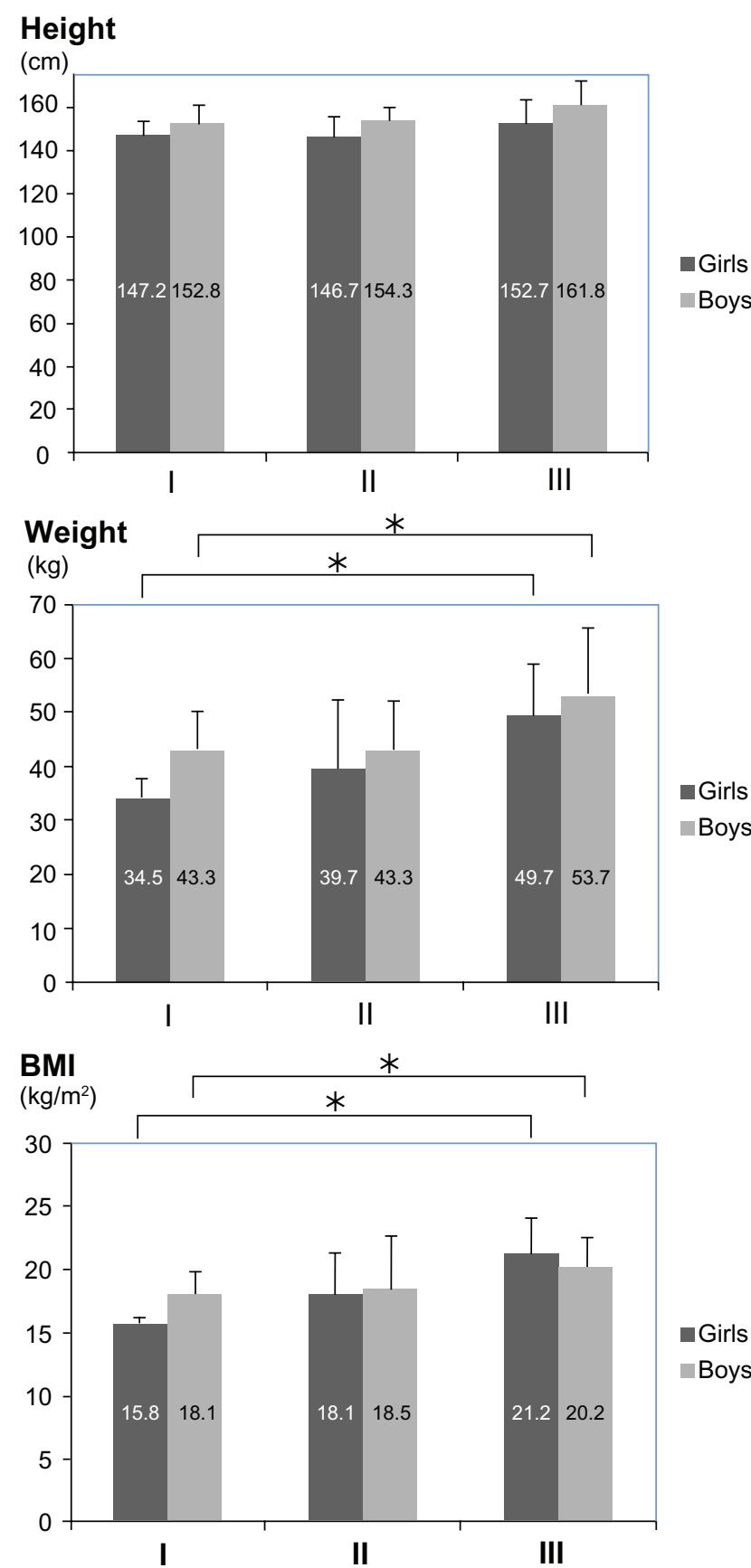

Figure 2 Relationship between height, weight, body mass index (BMI), and severity.

Notes: There was no significant difference in the mean height between severity I, II, and III in boys and girls groups. The body weight and body mass index were significantly less in boys and girls groups with severity of I than with severity of III by the Mann-Whitney $U$ test $(* P<0.05)$.
One of the limitations of this study is that this research is not longitudinal. Another limitation is that OSD was determined primarily by swelling and tenderness of the tibial tuberosity. In addition, the severity of OSD was evaluated by radiography only. In future longitudinal research, evaluation by MRI may increase the accuracy of results.

\section{Disclosure}

The authors report no conflicts of interest in this work.

\section{References}

1. Osgood RB. Lesions of the tibial tubercle occurring during adolescence. Boston Med Surg J. 1903;148:114-119.

2. Schlatter C. Verletzungen des scnabelformigen Fortsatzes der oberen Tibiaepiphyse. Brun Beitr Klin Chir. 1903;38:874-887.

3. Pease CN. Relationship of Streptococcus viridans to apophysitis of tibial tubercle. Am J Surg. 1934;24:149-150.

4. Cole JP. A study of Osgood-Schlatter disease. Surg Gynecol Obstet. 1937;65:55-67.

5. Ogden JA, Southwick WO. Osgood-Schlatter's disease and tibial tuberosity development. Clin Orthop. 1976;116:180-189.

6. Ogden JA, Tross RB, Murphy MJ. Fractures of the tibial tuberosity in adolescents. J Bone Joint Surg Am. 1980;62(2):205-215.

7. Ogden JA. Radiology of postnatal skeletal development. X. Patella and Tibial tuberosity. Skeletal Radiol. 1984;11(4):246-257.

8. Hirano A, Fukubayashi T, Ishii T, Ochiai N. Magnetic resonance imaging of Osgood-Schlatter disease: the course of the disease. Skeletal Radiol. 2002;31(6):334-342.

9. Gholve PA, Scher DM, Khakharia S, Widmann RF, Green DW. Osgood Schlatter syndrome. Curr Opin Pediatr. 2007;19(1):44-50.

10. Flowers MJ, Bhadreshwar DR. Tibial tuberosity excision for symptomatic Osgood-Schlatter disease. J Pediatr Orthop. 1995;15(3):292-297.

11. Blankstein A, Cohen I, Heiman Z, et al. Ultrasonography as a diagnostic modality and therapeutic adjuvant in the management of soft tissue foreign bodies in the lower extremities. Isr Med Assoc J. 2001; 3(6):411-413.

12. Ehrenborg G, Lagergren C. Roentgenologic changes in the OsgoodSchlatter lesion. Acta Chir Scand. 1961;121:315-327.

13. Kraus R, Berthold LD, Heiss C, Lässig M. Consecutive bilateral proximal tibial fractures after minor sports trauma. Eur J Pediatr Surg. 2009;19(1):41-43.
Open Access Journal of Sports Medicine

\section{Publish your work in this journal}

Open Access Journal of Sports Medicine is an international, peer-reviewed, open access journal publishing original research, reports, reviews and commentaries on all areas of sports medicine. The manuscript management system is completely online and includes a very quick and fair peer-review system.

\section{Dovepress}

Visit http://www.dovepress.com/testimonials.php to read real quotes from published authors. 